A

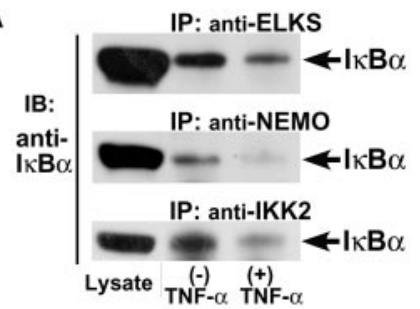

C
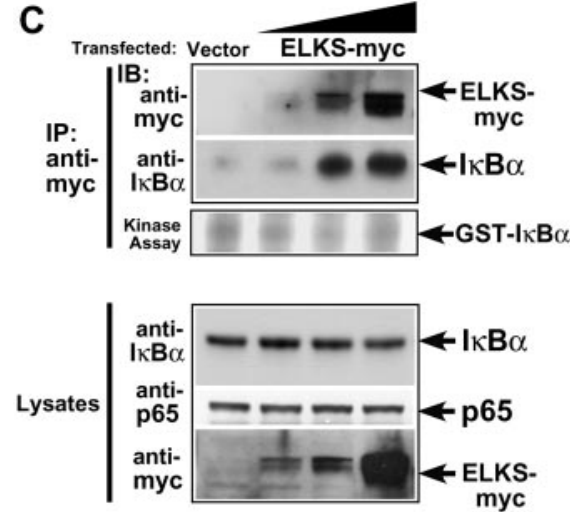

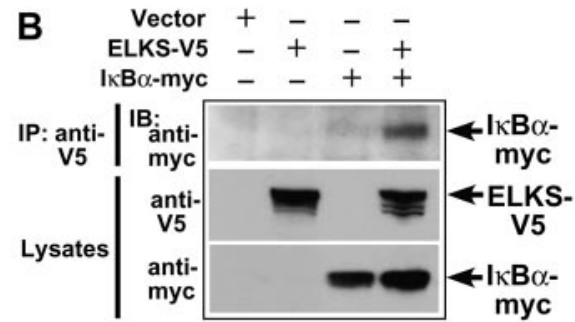

D
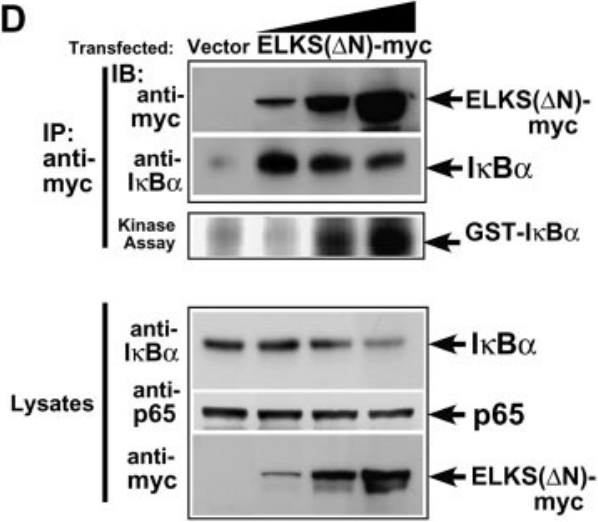

E

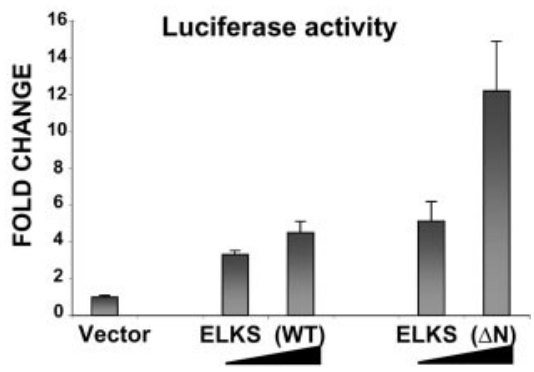

Fig. 4. ELKS recruits $I \kappa B \alpha$ and regulates IKK activity through modification of its $\mathrm{N}$-terminal domain. (A) Extracts from HeLa cells, untreated or treated with TNF- $\alpha$ for $7 \mathrm{~min}$, were immunoprecipitated with ELKS (N-terminal), NEMO, or IKK2 antisera. Immunoprecipitates and cell extracts $(50 \mu \mathrm{g})$ from each sample were immunoblotted with antibodies to IKB $\alpha$. (B) Extracts from 293T cells transfected with ELKS-V5, ІкB $\alpha-M y c$, or vector alone were immunoprecipitated with antibodies to V5. Immunoprecipitates and cell extracts $(50 \mu \mathrm{g})$ were immunoblotted with the indicated antibodies. Extracts from 293T cells transfected with vector alone or increasing amounts of (C) ELKS-Myc (wild type), or (D) $\operatorname{ELKS}(\Delta \mathrm{N})$-Myc (amino acids 1 to 134 deleted) (5 $\mu \mathrm{g}, 10 \mu \mathrm{g}$, and $20 \mu \mathrm{g}$, respectively) were immunoprecipitated with antibodies to Myc. The immunoprecipitates were split into two equal parts. Half the sample was resolved on SDS-PAGE and immunoblotted with antibodies to IкB $\alpha$ and Myc. The other half of the sample was assessed for IKK activity by an in vitro immune complex kinase assay with GST-IKB $\alpha(1-54)$ protein as substrate. (bottom) Cell extracts (50 $\mu g$ ) from each treated sample were resolved on SDS-PAGE and immunoblotted with the indicated antibodies. (E) HeLa cells were transfected with vector alone ( $3 \mu \mathrm{g})$, ELKS-Myc [wild type (WT)] (0.3 $\mu$ g and $3.0 \mu$ g, respectively), and ELKS $(\Delta \mathrm{N})-\mathrm{Myc}(0.3 \mu \mathrm{g}$ and $3.0 \mu \mathrm{g}$, respectively) in combination with an HIV-NF-KB luciferase reporter vector, and assessed for NF-kB-dependent transactivation 48 hours after transfection.

20. G. Tiscornia, O. Singer, M. Ikawa, I. M. Verma, Proc. Natl. Acad. Sci. U.S.A. 100, 1844 (2003).

21. T. Nakata, T. Yokota, M. Emi, S. Minami, Genes Chromosomes Cancer 35, 30 (2002)

22. H. L. Pahl, Oncogene 18, 6853 (1999).

23. D. J. Van Antwerp, S. J. Martin, T. Kafri, D. R. Green, J. M. Verma, Science 274, 787 (1996)

24. A. A. Beg, D. Baltimore, Science 274, 782 (1996).

25. C. Y. Wang, M. W. Mayo, A. S. Baldwin Jr., Science 274, 784 (1996)

26. Y. Yamamoto et al., J. Biol. Chem. 276, 36327 (2001).

27. C. Schmidt et al., Mol. Cell 12, 1287 (2003).

28. J. L. Ducut Sigala et al., unpublished data.

29. J. L. Poyet et al., J. Biol. Chem. 275, 37966 (2000).
30. E. D. Tang, N. Inohara, C. Y. Wang, G. Nunez, K. L. Guan, J. Biol. Chem. 278, 38566 (2003).

31. S. Tegethoff, J. Behlke, C. Scheidereit, Mol. Cell. Biol. 23, 2029 (2003).

32. We thank M. Emi for the gift of the ELKS $\alpha$ CDNA; and C. Larson, N. Somia, V. Tergoankar, R. Correa, A. Yanai, S. Withoff, P. Sigala, T. Cathomen, O. Singer, N. Tonnu, and N. Droin for helpful discussions, technical advice, and critical reading of the manuscript. I.M.V. is an American Cancer Society Professor of Molecular Biology. He is supported in part by grants from the $\mathrm{NIH}$, the Larry L. Hillblom Foundation, Inc., the Lebensfeld Foundation, the Wayne and Gladys Valley Foundation, and the $\mathrm{H}$. N. and Frances C. Berger
Foundation. J.L.D.S. is supported by the NIH National Institute of General Medical Sciences Minority Access to Research Careers predoctoral fellowship. V.B. is supported by Institut National de la Santé et de la Recherche Médicale (INSERM) and the Philippe Foundation Fellowships.

Supporting Online Material

www.sciencemag.org/cgi/content/full/304/5679/1963/ DC1

Materials and Methods

Figs. S1 and S2

References

26 March 2004; accepted 21 May 2004

\section{Computational Design of a Biologically Active Enzyme}

\author{
Mary A. Dwyer, ${ }^{1}$ Loren L. Looger, ${ }^{1 *}$ Homme W. Hellinga ${ }^{1} \dagger$
}

\begin{abstract}
Rational design of enzymes is a stringent test of our understanding of protein chemistry and has numerous potential applications. Here, we present and experimentally validate the computational design of enzyme activity in proteins of known structure. We have predicted mutations that introduce triose phosphate isomerase activity into ribosebinding protein, a receptor that normally lacks enzyme activity. The resulting designs contain 18 to 22 mutations, exhibit $10^{5}$ - to $10^{6}$-fold rate enhancements over the uncatalyzed reaction, and are biologically active, in that they support the growth of Escherichia coli under gluconeogenic conditions. The inherent generality of the design method suggests that many enzymes can be designed by this approach.
\end{abstract}

Enzymes are among the most proficient catalysts known (1), and they catalyze a wide variety of reactions in aqueous solutions under ambient conditions with exquisite selec- tivity and stereospecificity $(2,3)$. The rational design of enzymes has tremendous practical potential for developing novel synthetic biochemical pathways $(4,5)$, but presents a for- midable challenge and is one of the most stringent tests for understanding protein chemistry. Here, we present structure-based computational design techniques $(6,7)$ that predict mutations for the construction of catalytically active sites in proteins of known structure. Using these methods, we converted ribose-binding protein (8) into analogs (NovoTims) of the glycolytic enzyme triose phosphate isomerase (9). Several NovoTims exhibit rate enhancements of about $10^{5}$ to $10^{6}$ and are biologically active, as seen in their support of the growth of Escherichia coli under gluconeogenic conditions.

Triose phosphate isomerase (TIM) is an essential component of the Embden-Meyerhof pathway (10), interconverting dihydroxyacetone phosphate (DHAP) and glyceraldehyde 3phosphate (GAP) (Fig. 1A). In glycolysis, TIM channels these two triose phosphate products of aldolase into pyruvate; in gluconeogenesis, 
Fig. 1. Triose phosphate isomerase biochemistry. (A) Role of TIM in glycolysis (green arrows), gluconeogenesis (red arrows), and methylgloxal metabolism $(10,18)$ (G6P, glucose-6-phosphate; $F 1,6 P_{2}$, fructose1,6-bisphospate; PFK phosphofructokinase; MGS, methylglyoxal synthetase). (B) TIM mechanism. (C) Comparison of yeast TIM (16) (pink, flexible loop; red, catalytic residues; yellow, phospoglycolate) and RBP (8) (I and II, $\mathrm{N}$-terminal and $\mathrm{C}$-terminal domains, respectively; $\mathrm{H}$, hinge region; ribose, yellow) structures.
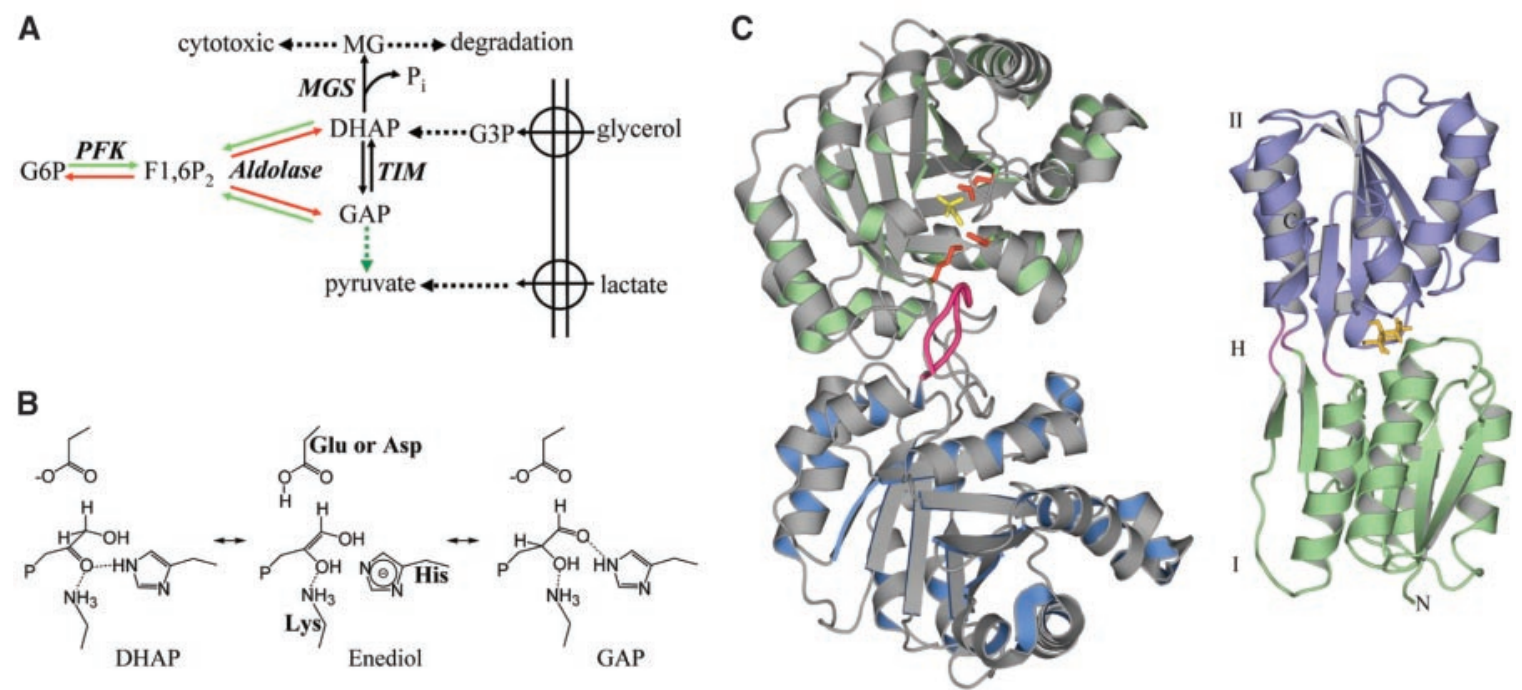

TIM ensures that both substrates are supplied to aldolase. The isomerization reaction involves two successive proton exchanges (9) (Fig. 1B) and is considered an archetype for proton transfer chemistry, which is central to many enzyme mechanisms (11). Extensive studies support a mechanism (9) whereby a carboxylate abstracts the DHAP pro- $R$ proton at $\mathrm{C} 1$ to form a cisenediol(ate) intermediate, followed by imidazole-mediated proton transfer between the $\mathrm{C} 1$ and $\mathrm{C} 2$ oxygens, yielding GAP. The $\mathrm{C} 1$ proton equilibrium constant $\left(\mathrm{p} K_{\mathrm{a}}\right.$ ) of $\sim 18$ imposes a large barrier to proton abstraction (12), which is overcome by a low-barrier hydrogen bond (13) that requires precise functional group alignment (14-16). Transition states are further stabilized electrostatically by lysine $(15,16)$. TIM also selects a substrate conformation that minimizes alignment of the enediolate double bond and phosphate $\pi$ systems, thereby stereoelectronically disfavoring an undesirable $\beta$-elimination of the phosphate (17) that produces methylglyoxal, which is cytotoxic in excess (18). A mobile loop permits substrate access and sequesters the reaction from solvent (19) (Fig. 1C). The TIM reaction therefore presents a complex design target demanding simultaneous capture of many mechanistic principles: acid-base catalysis, transition state stabilization, reactive group alignment, low-barrier hydrogen bonds, stereoelectronic control by ground state selection, electrostatic effects, and protein dynamics $(2,3)$.

Here, we demonstrate how structure-based computational design techniques can be used to introduce isomerase activity into the bacterial ribose-binding protein (RBP), a periplasmic receptor that has no known catalytic activity.

${ }^{1}$ Department of Biochemistry, Duke University Medical Center, Durham, NC 27710, USA.

*Present address: Department of Plant Biology, Carnegie Institute of Washington, Stanford, CA 94305, USA.

$\dagger$ To whom correspondence should be addressed. Email: hwh@biochem.duke.edu
RBP is a monomer and consists of two domains linked by a hinge region ( 8 ) (Fig. 1C). The protein adopts two conformations, a ligand-free open form, and a ligand-bound closed form, which interconvert via hinge-bending motions. Analogous to TIM, the ribose ligand is sequestered from solvent in the closed form. TIM is a homodimer of $\alpha / \beta$ barrel monomers $(15,16)$ (Fig. 1C). RBP and TIM structures fall into different topological classes; introduction of TIM activity into RBP is therefore equivalent to convergent evolution by computational design.

As a first step, we tested whether RBP can be redesigned to bind GAP and DHAP, regardless of catalysis. We used a recently developed algorithm (7) to predict mutations that convert RBP into a receptor for DHAP by changing the layer of residues directly contacting ribose in the wild-type protein structure. Sequences that form stereochemically complementary ligandbinding surfaces were identified using a combinatorial optimization algorithm (7) that integrates ligand docking and placement of amino side-chain rotamer libraries to locate energetic minima in a potential function incorporating van der Waals, hydrogen bonding, solvation, and electrostatic interactions (20) between the amino acids and ligand. Four designs (Fig. 2A) bind DHAP and GAP with micromolar affinities, as determined by titration of these substrates into fluorescent protein conjugates (21), but exhibit no TIM reactivity in colorimetric coupled enzyme assays (22). This experiment shows that RBP can be mutated to bind both substrates, which is a necessary preliminary finding before the introduction of catalysis.

To design catalytic activity, we developed a procedure that introduces catalytically active residues into the receptor design process. This approach is based on the premise that placement of a subset of reactive amino acid side chains within an active site in geometries compatible with bond formation to a substrate, tran- sition state, or reaction intermediate captures major contributions to reaction rate enhancement; the remainder of the binding surface is formed by residues selected on the basis of stereochemical complementarity between the protein and ligand, rather than explicit contributions to catalysis. The design process is divided into three parts (Fig. 3A). First, a geometrical definition of key interactions contributing to catalysis is generated (Fig. 3B). Second, a combinatorial search algorithm (6) identifies positions where placement of catalytic residues and substrate simultaneously satisfies these geometrical constraints. Third, the remainder complementary surface is generated around the placed substrate with the use of the receptor design algorithm (7). Designs were generated using geometrical relations between the enediolate reaction intermediate and the three catalytic residues (glutamate, histidine, lysine) as a minimalist model of interactions that are critical to catalysis. These definitions attempt to capture the dominant contributions to the reaction stereochemistry, without conserving nonessential features, such as the chirality of the proton abstraction from DHAP (pro- $R$ or pro- $S$ attack is allowed). As a consequence of the additional constraints imposed by explicitly specifying placement of the catalytic residues, the calculated sequences are distinct from those generated in the DHAP receptor designs (see above). We tested 14 designs subdivided into three families that differ in placement of the catalytic residues (Table 1); family 1 has the proton abstraction chirality that is the opposite of that from wild type, whereas families 2 and 3 retain the wild-type chirality. Seven designs show increases in GAP production over background. One design, NovoTim1.0, is significantly more active. It exhibits saturation kinetics [Table 2 (22)] and is competitively inhibited by phosphoglycolate [inhibition constant $\left.\left(K_{\mathrm{i}}\right)=130 \mu \mathrm{M}\right]$, a known inhibitor of wildtype $\operatorname{TIM}(9)\left(K_{\mathrm{i}}=4 \mu \mathrm{M}\right)$. 
Fig. 2. Predicted structures of RBP designs. (A) DHAP-binding receptor D1 (stereoview) with ligand (orange) and designed complementary surface residues (blue). (B) NovoTim1.0 with enediolate (orange), catalytic residues (red), and complementary surface (blue). (C) NovoTim 1.2 showing the layer of residues surrounding the active site (yellow), mutation of which confers near-wild type stability (enediolate, orange; catalytic residues, red; substrate-binding residues, blue). Also indicated are the mutations isolated by directed evolution of NovoTim 1.2 (orange cir-
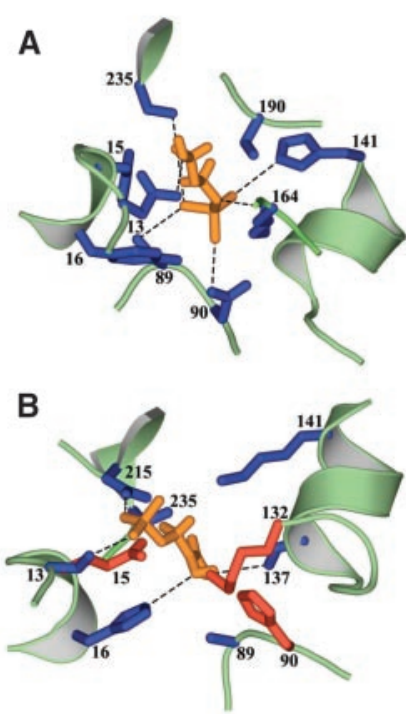
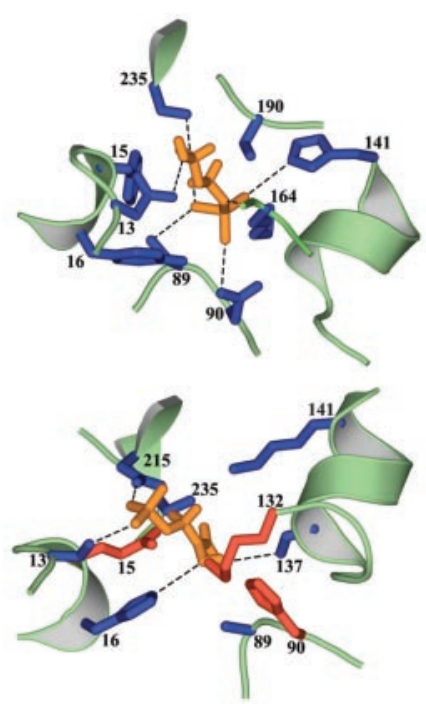

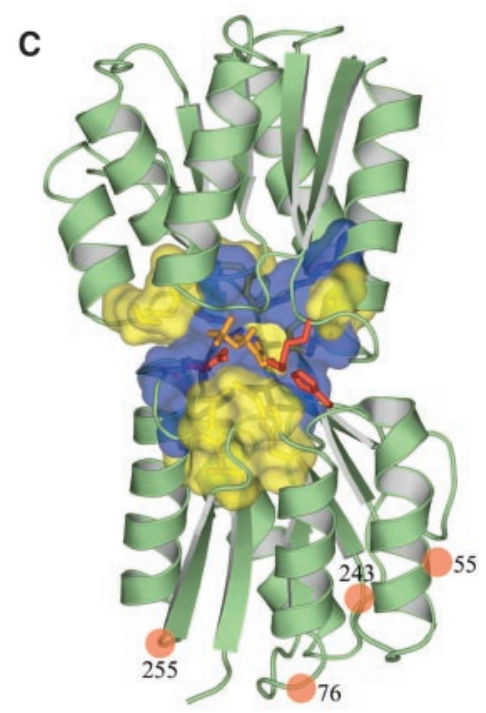
cles; view hides 264 at the hinge), that increase enzyme activity (Subscripts indicate the location of a residue position, as follows; I, N-terminal domain; II, C-terminal domain; $\mathrm{H}$, hinge. NovoTim1.2.1: Lys ${ }_{1}^{76}$ Asn, Lys ${ }^{243}{ }_{1}$ Ser; NovoTim1.2.2: Lys ${ }_{1}^{76}$ Ala, Glu ${ }^{255}{ }_{1}$ Val; NovoTim1.2.3: Asp ${ }^{264}{ }_{H}{ }^{G l n}$; NovoTim 1.2.4: Va ${ }^{155}{ }_{1}$ Ser).

NovoTim 1.0 is less thermostable than the parent protein (Fig. 4A). We postulated that steric imperfections in the interactions between the designed binding-surface residues and the surrounding protein matrix cause this decreased stability. Previously, we established that, in RBP-based metalloprotein designs, stability is restored by designing mutations in residue layers surrounding designed binding surfaces (23). We redesigned NovoTim1.0 in a similar manner (Fig. 2C; Table 1). In NovoTim1.1, the 13 original mutations were retained and 9 additional ones were introduced by computational design, which increased the stability by $5^{\circ} \mathrm{C}$. In NovoTim1.2, only the three catalytic residues were retained, and the sequences of the nine binding and nine interfacial residues were designed together. NovoTim1.2 stability is increased by $15^{\circ} \mathrm{C}$, approaching that of the parent protein. NovoTim1.1 has kinetic properties similar to those of NovoTim1.0, whereas in NovoTim1.2 the catalyzed reaction rate $\left(k_{\text {cat }}\right)$ and the Michaelis constant $\left(K_{\mathrm{M}}\right)$ are each improved about twofold (Table 2; Fig. 4B).

At least $95 \%$ of DHAP (GAP) is converted into GAP (DHAP) in the reaction catalyzed by the NovoTims, as judged by NADH $\left(\mathrm{NAD}^{+}\right.$) production (22). The loss of enzyme activity observed in single-, double-, and triple-alanine mutants of NovoTim1.2 indicates that all three designed catalytic residues make critical contributions to catalysis (Fig. 4C). The $\mathrm{pH}$ dependencies of the forward and reverse reactions catalyzed by NovoTim 1.2 are similar to those of wild-type TIM (Fig. 4D). These results show that the desired reaction is predominant, the designed catalytic groups are key to the enzyme mechanism,

Fig. 3. Computational enzyme design procedure. (A) Integration of the algorithms for placing side chains and ligands with predefined geometries (6) to generate partial sites that specify the location and structures of the catalytically active residues, with the design of stereochemically complementary substrate-binding surfaces (7) to design

A

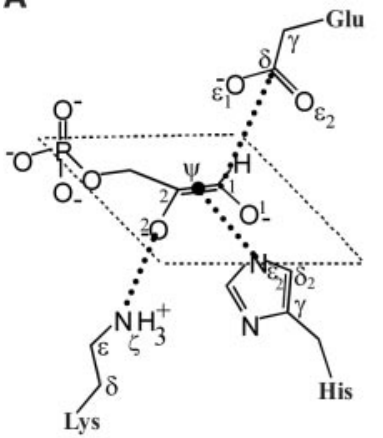

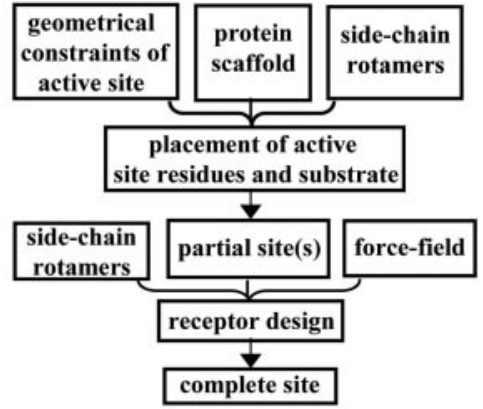
complete active sites.

(B) Geometric definition used to generate placement of the active site residues. Positioning of the catalytic residues (glutamate, histidine, lysine) is shown relative to the plane of the enediolate. The enediolate conformation is designed to minimize phosphate elimination and is derived from the structure of a phosphoglycolate complex (16). To define the constraints for histidine, a pseudoatom, $\psi$, was placed midway (circle) between $C_{1}$ and $C_{2}$. Geometrical constraints are formulated (6) in terms of intervals allowed for bond lengths $(l)$, angles $(\omega)$, and torsions $(\theta)$ for each residue relative to the enediolate: glutamate, $l\left(C_{1}, C_{\delta}: 2\right.$ to 5 $\AA) ; \omega_{1}\left(C_{\delta}, C_{1}, C_{2}: 107^{\circ} \pm 30^{\circ}\right) ; \omega_{2}\left(C_{1}, C_{2}, O_{\varepsilon 1}: 62.3^{\circ} \pm 30^{\circ}\right) ; \theta_{1}\left(O_{1}, C_{2}, C_{1}, C_{\delta}: 180^{\circ} \pm 15^{\circ}\right) ; \theta_{2}\left(C_{2}, C_{1}, C_{\delta}, O_{\varepsilon}\right.$ : unconstrained); $\theta_{3}\left(C_{1}, C_{\delta}, O_{\varepsilon 1}, O_{\varepsilon 2}: 0^{\circ} \pm 30^{\circ}\right)$; histidine: $l\left(N_{\varepsilon 2^{\prime}} \psi: 2\right.$ to $\left.4 \AA\right) ; \omega_{1}\left(C \gamma, N_{\varepsilon 2^{\prime}} \psi: 127.5^{\circ}\right) ; \omega_{2}\left(N_{\varepsilon 2^{\prime}}\right.$ $\left.\psi, C_{1}: 90^{\circ}\right) ; \theta_{1}\left(C_{\gamma^{\prime}}, C_{\delta 2}, N_{\varepsilon 2}, \psi: 180^{\circ}\right) ; \theta_{2}\left(C_{\delta 2}, N_{\varepsilon 2}, \psi, C_{1}: 0^{\circ} \pm 30^{\circ}\right) ; \theta_{3}\left(N_{\varepsilon 2}, \psi, C_{1}, O_{1}: 0^{\circ} \pm 45^{\circ}\right) ;$ lysine: l( $O_{2} 2^{\prime}$ $\mathrm{N}_{\zeta}: 2$ to $\left.5 \AA\right) ; \omega_{1}\left(C_{2}, \mathrm{O}_{2}, \mathrm{~N}_{\zeta}: 90^{\circ}\right.$ to $\left.180^{\circ}\right) ; \omega_{2}\left(\mathrm{O}_{2}, \mathrm{~N}_{\zeta}, \mathrm{C}_{\varepsilon}: 90^{\circ}\right.$ to $\left.180^{\circ}\right) ; \theta_{1}\left(\mathrm{C}_{\gamma^{\prime}}, \mathrm{C}_{2^{\prime}}, \mathrm{O}_{2}, \mathrm{~N}_{\zeta}: 180^{\circ} \pm 90^{\circ}\right) ; \theta_{2}\left(\mathrm{C}_{2^{\prime}}\right.$ $\mathrm{O}_{2}, \mathrm{~N}_{\zeta}, \mathrm{C}_{\varepsilon}$ : unconstrained); $\theta_{3}\left(\mathrm{O}_{2}, \mathrm{~N}_{\zeta}, \mathrm{C}_{\varepsilon}, \mathrm{C}_{\delta}\right.$ : unconstrained).

and the active site microenvironment approximates the naturally evolved enzyme.

In $E$. coli, gluconeogenic growth on lactate or glycerol requires TIM activity (Fig. 1A). Glycerol feeds into DHAP and places more stringent demands than lactate on TIM activity, because elevated DHAP levels increase cytotoxic methylglyoxal production, which is mitigated through TIM-mediated conversion of DHAP into GAP (18). Complementation of a TIM-deficient strain (10), DF502, by overexpressed NovoTims was tested on both gluconeogenic substrates (24) in the presence and absence of the inducer isopropyl- $\beta-D-$ thiogalactopyranoside (IPTG). NovoTims1.0 and 1.2 (1.1 not tested) support IPTGdependent growth on lactate, but not glycerol. NovoTim 1.2 was further mutagenized by an error-prone polymerase chain reaction (25), and mutants were selected on glycerol. Four isolates were obtained from about $10^{5}$ transformants. The different mutations in NovoTims1.2.1 to 1.2.4 are localized on the protein surface (Fig. 2C) and improve $k_{\text {cat }}$ and $K_{\mathrm{M}}$ values, with the largest changes corresponding to twofold and threefold increases in $k_{\text {cat }}$ and $k_{\text {cat }} / K_{\mathrm{M}}$ values, respectively.

We have successfully converted a protein devoid of catalytic activity into a triose phos- 
Table 1. Sequences of the designs. Superscripts indicate residue sequence (31) (Fig.1C); lowercase, wild-type residue retained by the calculation; boldface, catalytic residues; underline, side chains make hydrogen bonds with substrate. Protein (column 1), DHAP- and GAP-binding receptors, D.1 to D.4; first-round designed enzymes (NovoTims) are divided into three families, Ntim1(b to e), 2(a to e), and 3(a to d). NovoTim1.0 is the most active first-round design. The distances of the modeled catalytic residues approximate the wild-type values (atom names as in Fig. 3). C1 to nearest carboxylate oxygen: wild type, $2.5 \AA$; family $1,2.9 \AA$; family $2,3.0 \AA$; family $3,3.1 \AA$. $C 1$ to imidazole $\mathrm{N}_{\varepsilon}$ : wild type, $3.3 \AA$; family $1,3.6 \AA$; family $2,3.6 \AA$; family 3, $3.6 \AA$. Lysine $N_{\zeta}$ to O2: wild type, $3.2 \AA$; family $1,4.5 \AA$; family $2,3.1 \AA$; family 3 , $3.0 \AA$. Activity (last column) from a semiquantitative assessment of activity based on end-point analysis of the forward (DHAP to GAP) reaction: -, background (uncatalyzed);,$+>5$-fold increase after 6 hours;,$+++>10$-fold after $5 \mathrm{~min} ;++++$, $>20$-fold increase after 5 min.

\begin{tabular}{|c|c|c|c|c|c|c|c|c|c|c|c|c|c|c|c|c|}
\hline \multirow{2}{*}{$\frac{\text { Protein }}{\text { D.1 }}$} & \multicolumn{15}{|c|}{ Binding surface residues } & \multirow{2}{*}{$\frac{\text { Activity }}{-}$} \\
\hline & $\underline{N}^{13}$ & $\underline{Y}^{15}$ & $f^{16}$ & $\underline{S}^{89}$ & $\underline{\mathrm{N}}^{90}$ & $G^{137}$ & $\underline{H}^{141}$ & $\underline{\mathrm{K}}^{164}$ & $\underline{\mathrm{S}}^{190}$ & $G^{215}$ & $\mathrm{q}^{235}$ & & & & & \\
\hline D.2 & $\overline{\mathrm{N}}^{13}$ & $\underline{\overline{\mathrm{H}}}^{15}$ & $f^{16}$ & $\mathrm{G}^{89}$ & $\mathrm{~K}^{90}$ & $\underline{S}^{137}$ & $\underline{\mathrm{K}}^{141}$ & $\underline{\mathrm{K}}^{164}$ & $\underline{\mathrm{S}}^{190}$ & $\underline{s}^{215}$ & $\underline{M}^{235}$ & & & & & - \\
\hline D. 3 & $\overline{\mathrm{K}^{13}}$ & $\mathrm{f}^{15}$ & $\mathrm{~L}^{16}$ & $G^{89}$ & $\mathrm{~S}^{90}$ & $\bar{S}^{137}$ & $\bar{M}^{141}$ & $\overline{\mathrm{K}}^{164}$ & $\overline{\mathrm{T}}^{190}$ & $\mathrm{~A}^{215}$ & $\mathrm{~V}^{235}$ & & & & & - \\
\hline D.4 & $\overline{\mathrm{K}}^{13}$ & $f^{15}$ & $\mathrm{Q}^{16}$ & $\underline{S}^{89}$ & $\underline{\underline{S}}^{90}$ & $\underline{\underline{S}}^{137}$ & $\overline{\mathrm{K}}^{141}$ & $\bar{L}^{164}$ & $\overline{\underline{S}}^{190}$ & $A^{215}$ & $q^{235}$ & & & & & - \\
\hline NTim1.0 & $\overline{\mathrm{S}}^{13}$ & $\underline{\mathbf{E}}^{15}$ & $\overrightarrow{\mathrm{H}}^{16}$ & $\bar{A}^{89}$ & $\underline{\underline{\mathbf{H}}}^{90}$ & $\underline{\mathbf{K}}^{132}$ & $\overline{\underline{S}}^{137}$ & $\underline{\mathrm{K}}^{141}$ & $\overline{\mathrm{G}}^{164}$ & $G^{190}$ & $\underline{N}^{192}$ & $f^{214}$ & $\underline{s}^{215}$ & $q^{235}$ & & +++ \\
\hline $1 \mathrm{~b}$ & $\mathrm{~S}^{13}$ & $\overline{\mathbf{E}}^{15}$ & $\mathrm{f}^{16}$ & $A^{89}$ & $\overline{\mathbf{H}}^{90}$ & $\overline{\mathbf{K}}^{132}$ & $\mathrm{~S}^{137}$ & $\overline{\mathrm{K}}^{141}$ & $G^{164}$ & $\mathrm{G}^{190}$ & $\mathrm{E}^{192}$ & $f^{214}$ & $\mathrm{~A}^{215}$ & $\mathrm{~T}^{235}$ & & + \\
\hline $1 \mathrm{c}$ & $\underline{\mathrm{S}}^{13}$ & $\underline{\mathbf{E}}^{15}$ & $\underline{\mathrm{H}}^{16}$ & $A^{89}$ & $\underline{\mathbf{H}}^{90}$ & $\overline{\mathbf{K}}^{132}$ & $S^{137}$ & $\overline{\overline{\mathrm{K}}}^{141}$ & $G^{164}$ & $G^{190}$ & $\underline{N}^{192}$ & $f^{214}$ & $\underline{T}^{215}$ & $q^{235}$ & & + \\
\hline $1 d$ & $\underline{S}^{13}$ & $\underline{\mathbf{E}}^{15}$ & $\underline{H}^{16}$ & $A^{89}$ & $\underline{\mathbf{H}}^{90}$ & $\overline{\mathbf{K}}^{132}$ & $S^{137}$ & $\overline{\overline{\mathrm{K}}}^{141}$ & $G^{164}$ & $G^{190}$ & $\mathrm{E}^{192}$ & $f^{214}$ & $\overline{\bar{T}}^{215}$ & $S^{235}$ & & + \\
\hline $1 \mathrm{e}$ & $\underline{\mathrm{S}}^{13}$ & $\underline{\mathbf{E}}^{15}$ & $\underline{\mathrm{H}}^{16}$ & $A^{89}$ & $\underline{\mathbf{H}}^{90}$ & $\underline{\mathbf{K}}^{132}$ & $\mathrm{~S}^{137}$ & $\underline{\overline{\mathrm{K}}}^{141}$ & $G^{164}$ & $G^{190}$ & $\underline{\bar{E}}^{192}$ & $f^{214}$ & $\underline{\bar{s}}^{215}$ & $q^{235}$ & & + \\
\hline $2 a$ & $\bar{S}^{9}$ & $\overline{\mathbf{E}}^{10}$ & $\overline{\mathrm{H}}^{13}$ & $f^{15}$ & $\overline{\mathrm{K}^{16}}$ & $\overline{S^{41}}$ & $S^{64}$ & $\bar{d}^{89}$ & $\mathbf{H}^{90}$ & $\mathrm{~K}^{132}$ & $\overline{\mathbf{K}}^{137}$ & $\mathrm{~K}^{141}$ & $\overline{\mathrm{G}}^{164}$ & $\mathrm{G}^{190}$ & $A^{215}$ & - \\
\hline $2 b$ & $\underline{\mathrm{S}}^{9}$ & $\underline{\mathbf{E}}^{10}$ & $\underline{\mathrm{H}}^{13}$ & $f^{15}$ & $\underline{\mathrm{K}}^{16}$ & $\underline{\mathrm{S}}^{41}$ & $\underline{\underline{S}}^{64}$ & $\underline{\bar{d}}^{89}$ & $\underline{\mathbf{H}}^{90}$ & $\underline{\mathrm{K}}^{132}$ & $\underline{\mathbf{K}}^{137}$ & $\underline{\overline{\mathrm{K}}}^{141}$ & $G^{164}$ & $G^{190}$ & $\mathrm{~L}^{215}$ & + \\
\hline $2 c$ & $\underline{\underline{S}}^{9}$ & $\underline{\mathbf{E}}^{10}$ & $\underline{\mathrm{S}}^{13}$ & $\mathrm{Q}^{15}$ & $\underline{\mathrm{S}}^{16}$ & $\underline{\mathrm{S}}^{41}$ & $\underline{\mathrm{K}}^{64}$ & $\mathrm{~A}^{89}$ & $\underline{\mathbf{H}}^{90}$ & $\underline{\mathrm{K}}^{132}$ & $\underline{\mathbf{K}}^{137}$ & $\underline{\mathrm{K}}^{141}$ & $G^{164}$ & $G^{190}$ & $A^{215}$ & - \\
\hline $2 d$ & $\mathrm{G}^{9}$ & $\mathbf{E}^{10}$ & $\mathrm{H}^{13}$ & $f^{15}$ & $\mathrm{~K}^{16}$ & $\bar{K}^{41}$ & $\mathrm{~S}^{64}$ & $d^{89}$ & $\mathbf{H}^{90}$ & $\mathrm{~K}^{132}$ & $\mathbf{K}^{137}$ & $\overline{\mathrm{K}}^{141}$ & $G^{164}$ & $\mathrm{G}^{190}$ & $A^{215}$ & _- \\
\hline $2 e$ & $G^{9}$ & $\underline{\mathbf{E}}^{10}$ & $\underline{\mathrm{H}}^{13}$ & $f^{15}$ & $\underline{\overline{\mathrm{K}}}^{16}$ & $\underline{\mathrm{S}}^{41}$ & $\underline{S}^{64}$ & $\underline{\mathrm{d}}^{89}$ & $\underline{\mathbf{H}}^{90}$ & $\underline{\mathrm{K}}^{132}$ & $\underline{\mathbf{K}}^{137}$ & $\underline{\mathrm{K}}^{141}$ & $G^{164}$ & $G^{190}$ & $A^{215}$ & - \\
\hline $3 a$ & $S^{9}$ & $\overline{\underline{T}}^{10}$ & $\mathrm{~A}^{13}$ & $\mathbf{H}^{16}$ & $\mathrm{~T}^{64}$ & $\bar{W}^{89}$ & $\overline{\mathrm{H}}^{90}$ & $\overline{\mathrm{V}}^{132}$ & $\mathbf{E}^{135}$ & $\overline{\mathrm{G}}^{137}$ & $\underline{S}^{138}$ & $\overline{\mathrm{K}}^{141}$ & $G^{164}$ & $L^{189}$ & $\mathbf{K}^{190}$ & + \\
\hline $3 b$ & $\underline{\underline{S}}^{9}$ & $\overline{\mathrm{T}}^{10}$ & $A^{13}$ & $\overline{\mathbf{H}}^{16}$ & $\overline{\mathrm{T}}^{64}$ & $W^{89}$ & $\overline{\mathrm{H}}^{90}$ & $\mathrm{~V}^{132}$ & $\mathbf{E}^{135}$ & $\mathrm{G}^{137}$ & $\mathrm{~S}^{138}$ & $\mathrm{~K}^{141}$ & $\mathrm{G}^{164}$ & $L^{189}$ & $\overline{\mathbf{K}}^{190}$ & - \\
\hline $3 c$ & $\mathrm{~S}^{9}$ & $\overline{\mathrm{T}}^{10}$ & $A^{13}$ & $\mathbf{H}^{16}$ & $\bar{D}^{64}$ & $W^{89}$ & $\bar{H}^{90}$ & $\mathrm{~V}^{132}$ & $\bar{E}^{135}$ & $G^{137}$ & $\mathrm{~S}^{138}$ & $\overline{\mathrm{K}}^{141}$ & $G^{164}$ & $L^{189}$ & $\overline{\mathbf{K}}^{190}$ & - \\
\hline $3 d$ & $\underline{S}^{9}$ & $\underline{\overline{\mathrm{T}}}^{10}$ & $A^{13}$ & $\underline{\mathbf{H}}^{16}$ & $\bar{M}^{64}$ & $W^{89}$ & $\underline{\bar{H}}^{90}$ & $\mathrm{~V}^{132}$ & $\overline{\bar{E}}^{135}$ & $G^{137}$ & $\underline{\mathrm{S}}^{138}$ & $\underline{\mathrm{K}}^{141}$ & $G^{164}$ & $\mathrm{~L}^{189}$ & $\overline{\mathbf{K}}^{190}$ & - \\
\hline NTim1.1* & $\underline{\mathrm{S}}^{13}$ & $\underline{\overline{\mathbf{E}}}^{15}$ & $\underline{\mathrm{H}}^{16}$ & $\underline{A}^{89}$ & $\underline{\mathbf{H}}^{90}$ & $\underline{\mathbf{K}}^{132}$ & $\underline{\underline{S}}^{137}$ & $\underline{\mathrm{K}}^{141}$ & $\mathrm{G}^{164}$ & $G^{190}$ & $\underline{\underline{N}}^{192}$ & $f^{214}$ & $\underline{s}^{215}$ & $q^{235}$ & & +++ \\
\hline NTim1.2* & $\underline{\underline{S}}^{13}$ & $\underline{\underline{\mathbf{E}}}^{15}$ & $\underline{\overline{\mathrm{H}}}^{16}$ & $\underline{\mathrm{D}}^{89}$ & $\underline{\underline{\mathbf{H}}}^{90}$ & $\underline{\mathbf{K}}^{132}$ & $\underline{\underline{S}}^{137}$ & $\underline{\overline{\mathrm{K}}}^{141}$ & $G^{164}$ & $G^{190}$ & $\underline{\underline{E}}^{192}$ & $f^{214}$ & $\underline{N}^{215}$ & $\underline{\underline{s}}^{235}$ & & ++++ \\
\hline
\end{tabular}

*NovoTim 1.1 and NovoTim1.2 are improvements based on NovoTim, with a secondary layer of residues (included in the calculation, but not tabulated: $1.1, I^{8}, \mathrm{H}^{10}, \mathrm{I}^{19}, \mathrm{~T}^{64}, \mathrm{Y}^{105}$, $\left.A^{138}, \underline{K}^{217}, \underline{r}^{166}, l^{265} 1.2, I^{8}, \underline{N}^{10}, l^{19}, \underline{H}^{64}, \underline{Y}^{105}, \underline{\underline{1}}^{135}, A^{138}, \underline{\underline{1}}^{166}, \underline{L}^{265}\right)$.

Fig. 4. Properties of selected designs. (A) Thermostability (reported as midpoint melting transition temperature, $T_{\text {m, }}$ values) monitored by temperature dependence of ellipticity (28) (wild-type RBP $(\diamond)$, $T_{m}=58^{\circ} \mathrm{C} ;$ NovoTim 1.0 (豆), $T_{\mathrm{m}}=37^{\circ} \mathrm{C}$; Novo$\operatorname{Tim} 1.1(\diamond), T_{m}=43^{\circ} \mathrm{C}$; NovoTim1.2, $(\bullet), T_{m}$ $=52^{\circ} \mathrm{C}$ ). (B) Steadystate kinetics [Lineweaver-Burke transformation (29)] of NovoTim1.2 for forward (DHAP to GAP, top) and reverse (GAP to DHAP, bottom) reactions. (C) Alanine mutants of catalytic residues $\left(\mathrm{Glu}^{15}\right.$, His $^{90}$, Lys ${ }^{132}$ ) in NovoTim1.2, presented as energy difference diagrams (30) [effects on rate enhancements $\left(k_{\text {cat }}\right.$ changes), stippled; effects on

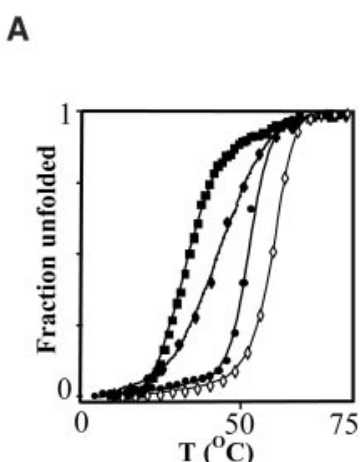

B
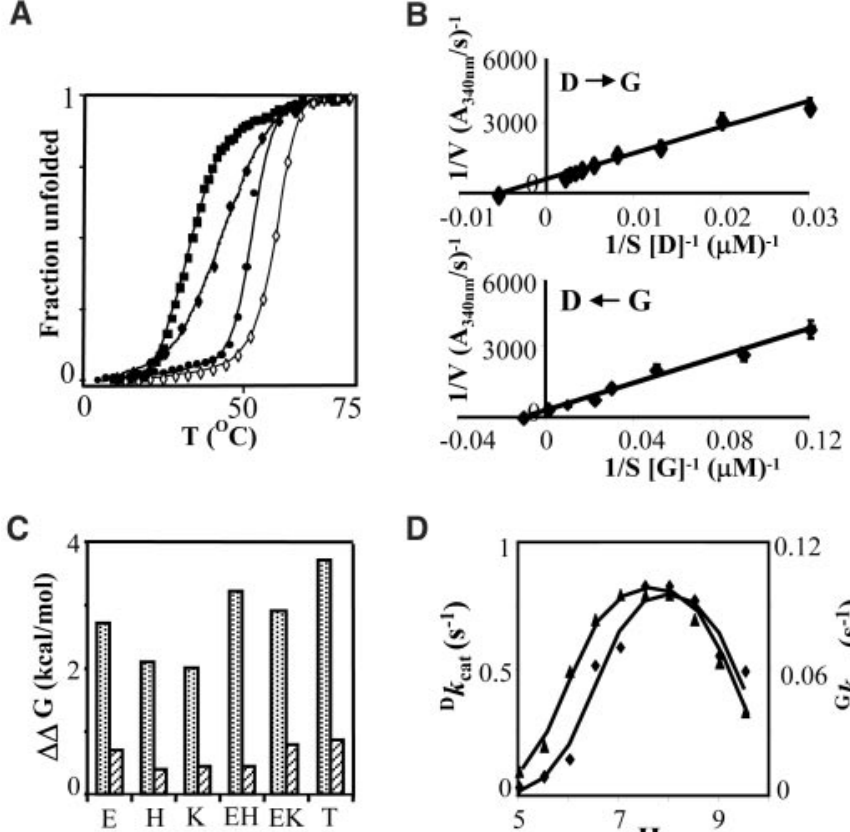

D

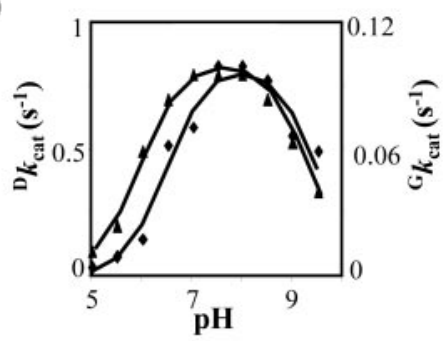

Michaelis constant ( $K_{M}$ changes), hashed]. (D) pH dependence of $k_{\text {cat }}$ for the forward $\left({ }^{(} k_{\text {cat }}, \mathbf{\Delta}\right)$ and reverse $\left({ }^{c} k_{\text {cat }} \diamond\right)$ reactions of NovoTim1.2 [calculated app $K_{a}$ values: forward $(6.5,9.5)$; reverse $(5.9,9.3)$ ].

phate isomerase, using computational design techniques to predict 13 to 21 mutations that introduce three catalytically active residues together with a stereochemically complementary substrate-binding surface. This minimalist design is based on key short-range interactions observed in naturally evolved TIMs and is sufficient to increase the NovoTim-catalyzed reac- tion $10^{5}$ - to $10^{6}$-fold over background. This rate enhancement is the largest so far as we know reported for rationally designed enzymes $(4,5)$. NovoTim1.2 is sufficiently active to support growth under permissive gluconeogenic conditions and requires only small improvements to support full biological activity. Nevertheless, the $k_{\text {cat }}$ and $k_{\text {cat }} / K_{\mathrm{M}}$ values of NovoTim1.2.1 are less than wild-type TIM, by factors of 2700 and 220, respectively; the apparent second-order rate constant of wild-type TIM approaches the diffusion-limited encounter of enzyme with substrate (9). Alanine-scanning mutagenesis indicates that all residues designed to be catalytically active contribute substantially to rate enhancement. Furthermore, the electrostatic microenvironment as probed by $\mathrm{pH}$ dependence of $k_{\text {cat }}$ is similar to that of the wild-type enzyme. However, it is likely that NovoTims have a suboptimal hydrogen bond between the catalytic glutamate and substrate $\mathrm{C} 1$ proton, which is a critical feature of the TIM reaction mechanism (14-16). [We note that shortening of glutamate to aspartate in the wild-type enzyme (26), presumably destroying the low-barrier hydrogen bonds, results in a mutant with activity similar to that of NovoTims.] Elaboration of the minimalist mechanism in future designs will allow testing of other contributions to rate enhancement, such as protein dynamics and long-range electrostatics.

The combined placement of mechanistically critical residues with construction of a surface that is stereochemically complementary to the entire substrate (and product) is a critical aspect of the design method presented here. This capability was absent in previously reported attempts at enzyme design (4) and is likely to be the main reason for the much higher rate enhancements and apparent second-order rate constants observed in this study. With the prediction accuracies now within reach of computational protein design $(7,27)$ and with the introduction of increasing levels of mechanistic detail and sophistication in future designs, we 
Table 2. Kinetic parameters for forward and reverse isomerization reactions. $k_{\text {uncat, }}$ uncatalyzed reaction rate from (32); ${ }^{\text {app }} K_{\text {eq }}$ (last column) is the apparent equilibrium constant [Haldane constant (30)] calculated from the ratio $k_{\mathrm{cat}} / K_{\mathrm{M}}$ values of the forward and reverse reactions measured at $25^{\circ} \mathrm{C}, 100$ $\mathrm{mM}$ triethanolamine, $\mathrm{pH} 7.8(9,22)$. nd, not determined. wtTIM, wild-type parameters from (9).

\begin{tabular}{|c|c|c|c|c|c|c|c|c|c|}
\hline \multirow[b]{2}{*}{ Protein } & \multicolumn{4}{|c|}{ DHAP $\rightarrow$ GAP } & \multicolumn{4}{|c|}{$\mathrm{DHAP} \leftarrow \mathrm{GAP}$} & \multirow[b]{2}{*}{${ }^{\text {app }} K_{\text {eq }}$} \\
\hline & $\begin{array}{c}k^{\mathrm{cat}} \\
\left(\mathrm{s}^{-1}\right)\end{array}$ & $\begin{array}{c}K_{M} \\
(\mu M)\end{array}$ & $\begin{array}{c}k_{\mathrm{cat}} / K_{\mathrm{M}} \\
\left(\mathrm{M}^{-1} \mathrm{~s}^{-1}\right)\end{array}$ & $k_{\text {cat }} / k_{\text {uncat }}$ & $\begin{array}{l}k_{\mathrm{cat}} \\
\left(\mathrm{s}^{-1}\right)\end{array}$ & $\begin{array}{c}K_{M} \\
(\mu \mathrm{M})\end{array}$ & $\begin{array}{c}k_{\mathrm{cat}} / K_{\mathrm{M}} \\
\left(\mathrm{M}^{-1} \mathrm{~s}^{-1}\right)\end{array}$ & $k_{\text {cat }} / k_{\text {uncat }}$ & \\
\hline \multicolumn{10}{|l|}{ NovoTim } \\
\hline 1.0 & 0.05 & 330 & $1.5 \times 10^{2}$ & $2.4 \times 10^{5}$ & nd & nd & nd & nd & nd \\
\hline 1.2 & 0.1 & 180 & $5.6 \times 10^{2}$ & $5.0 \times 10^{5}$ & 0.8 & 92 & $8.6 \times 10^{3}$ & $1.8 \times 10^{5}$ & 15 \\
\hline 1.2 .1 & 0.18 & 140 & $1.4 \times 10^{3}$ & $9.0 \times 10^{5}$ & 1.5 & 85 & $1.7 \times 10^{4}$ & $3.4 \times 10^{5}$ & 12 \\
\hline 1.2 .2 & 0.14 & 165 & $8.2 \times 10^{2}$ & $7.0 \times 10^{5}$ & 1.2 & 89 & $1.4 \times 10^{4}$ & $2.7 \times 10^{5}$ & 17 \\
\hline 1.2 .3 & 0.17 & 100 & $1.8 \times 10^{3}$ & $8.5 \times 10^{5}$ & 1.2 & 103 & $1.2 \times 10^{4}$ & $2.7 \times 10^{5}$ & 7 \\
\hline 1.2 .4 & 0.11 & 105 & $1.0 \times 10^{3}$ & $5.5 \times 10^{5}$ & 1.1 & 51 & $2.1 \times 10^{4}$ & $2.5 \times 10^{5}$ & 21 \\
\hline wtTIM & 487 & 1600 & $3.0 \times 10^{5}$ & $>1 \times 10^{9}$ & 4. $\times 10^{3}$ & 390 & $1.0 \times 10^{7}$ & $1.0 \times 10^{9}$ & 33 \\
\hline
\end{tabular}

anticipate that the design method will be extended to other substrates and reactions.

\section{References and Notes}

1. R. Wolfenden, M. J. Snider, Acc. Chem. Res. 34, 938 (2001).

2. S. J. Benkovic, S. Hammes-Schiffer, Science 301, 1196 (2003).

3. M. Garcia-Viloca, J. Gao, M. Karplus, D. G. Truhlar, Science 303, 186 (2004)

4. D. N. Bolon, C. A. Voigt, S. L. Mayo, Curr. Opin. Chem. Biol. 6, 125 (2002)

5. D. Hilvert, Annu. Rev. Biochem. 69, 751 (2000).

6. H. W. Hellinga, F. M. Richards, J. Mol. Biol. 222, 763 (1991)

7. L. L. Looger, M. A. Dwyer, J. J. Smith, H. W. Hellinga, Nature 423, 185 (2003).

8. S. L. Mowbray, L. B. Cole, J. Mol. Biol. 225, 155 (1992)

9. J. R. Knowles, Nature 350, 121 (1991).

10. D. G. Fraenkel, Annu. Rev. Biochem. 55, 317 (1986)

11. J. P. Richard, T. L. Amyes, Curr. Opin. Chem. Biol. 5 626 (2001)

12. J. P. Richard, Biochemistry 24, 949 (1985)

13. W. W. Cleland, P. A. Frey, J. A. Gerlt, J. Biol. Chem. 273, 25529 (1998).
14. T. K. Harris, C. Abeygunawardana, A. S. Mildvan, Biochemistry 36, 14661 (1997).

15. I. Kursula, R. K. Wierenga, J. Biol. Chem. 278, 9544 (2003).

16. G. Jogl, S. Rozovsky, A. E. McDermott, L. Tong, Proc. Natl. Acad. Sci. U.S.A. 100, 50 (2003).

17. E. Lolis, G. A. Petsko, Biochemistry 29, 6619 (1990).

18. G. P. Ferguson, S. Totemeyer, M. J. MacLean, I. R. Booth, Arch. Microbiol. 170, 209 (1998).

19. N. S. Sampson, J. R. Knowles, Biochemistry 31, 8488 (1992).

20. M. S. Wisz, H. W. Hellinga, Proteins 51, 360 (2003)

21. R. M. De Lorimier et al., Protein Sci. 11, 2655 (2002)

22. Information on materials and methods is available on Science Online.

23. M. A. Dwyer, L. L. Looger, H. W. Hellinga, Proc. Natl. Acad. Sci. U.S.A. 100, 11255 (2003).

24. J. D. Hermes, S. M. Parekh, S. C. Blacklow, H. Koster, J. R. Knowles, Gene 84, 143 (1989).

25. M. Zaccolo, D. M. Williams, D. M. Brown, E. Gherardi, J. Mol. Biol. 255, 589 (1996).

26. R. T. Raines, E. L. Sutton, D. R. Straus, W. Gilbert, J. R. Knowles, Biochemistry 25, 7142 (1986).

27. B. Kuhlman et al., Science 302, 1364 (2003).
28. J. A. Schellman, Compt. Rend. Trav. Lab. Carlsberg Ser. Chim. 29, 230 (1955)

29. I. H. Segel, Enzyme Kinetics (Wiley, New York, 1975)

30. A. Fersht, Structure and Mechanism in Protein Science: A Guide to Enzyme Catalysis and Protein Folding (Freeman, New York, 1999).

31. Single-letter abbreviations for the amino acid residues are as follows: A, Ala; C, Cys; D, Asp; E, Glu; F, Phe; G, Gly; H, His; I, Ile; K, Lys; L, Leu; M, Met; N, Asn; P, Pro; Q, Gln; R, Arg; S, Ser; T, Thr; V, Val; W, Trp; X, any amino acid; and $Y$, Tyr.

32. A. Hall, J. R. Knowles, Biochemistry 14, 4348 (1975).

33. We thank G. Hammes, M. Allert, and K. Midelfort for insightful discussion and L. Loew for the gift of the styryl dyes. This work was supported by a grant to H.W.H. from the Defense Advanced Research Projects Agency.

Supporting Online Material

www.sciencemag.org/cgi/content/full/304/5679/1967/DC 1

Materials and Methods

Figs. S1 to S4

Tables S1 and S2

References

29 March 2004; accepted 6 May 2004

\section{RNAi-Independent Heterochromatin Nucleation by the Stress-Activated ATF/CREB Family Proteins}

\author{
Songtao Jia, Ken-ichi Noma, Shiv I. S. Grewal*
}

\begin{abstract}
At the silent mating-type interval of fission yeast, the RNA interference (RNAi) machinery cooperates with cenH, a DNA element homologous to centromeric repeats, to initiate heterochromatin formation. However, in RNAi mutants, heterochromatin assembly can still occur at low efficiency. Here, we report that Atf1 and Pcr1, two ATF/CREB family proteins, act in a parallel mechanism to the RNAi pathway for heterochromatin nucleation. Deletion of atf1 or pcr 1 alone has little effect on silencing at the mating-type region, but when combined with RNAi mutants, double mutants fail to nucleate heterochromatin assembly. Moreover, deletion of atf 1 or $p c r 1$ in combination with cenH deletion causes loss of silencing and heterochromatin formation. Furthermore, Atf1 and Pcr 1 bind to the mating-type region and target histone $\mathrm{H} 3$ lysine- 9 methylation and the Swi6 protein essential for heterochromatin assembly. These analyses link ATF/CREB family proteins, involved in cellular response to environmental stresses, to nucleation of constitutive heterochromatin.
\end{abstract}

Heterochromatin governs diverse processes ranging from gene regulation and chromosome segregation to suppression of deleterious recombination in repetitive sequences. In the fission yeast Schizosaccharomyces pombe, heterochromatin is present mainly at the centromeres, telomeres, and a $20-\mathrm{kb}$ silent domain at the mating-type region, the loci that are preferentially enriched in histone $\mathrm{H} 3$ lysine9 (H3-K9) methylation and the Swi6 protein (a homolog of mammalian HP1 proteins) (1). The mechanisms that define these chromosomal regions as preferred sites of heterochromatin formation are not fully understood. Recent studies have implicated RNAi pathway in targeting of heterochromatin to repetitive DNA sequences in $S$. pombe and in other organisms including Tetrahymena, Arabidopsis, and Drosophila (2-7). It has been demonstrated that deletions of factors involved in the RNAi pathway such as Dicer $(d c r l)$, RNA-dependent RNA polymerase $(r d p 1)$, and Argonaute (agol) disrupt heterochromatin assembly at centromeres (4). Moreover, cenH sequence $[96 \%$ similar to $\mathrm{dg}$ and $\mathrm{dh}$ centromeric repeats (8)] that is present at the silent mating-type (mat $2 / 3$ ) region (see Fig. 1A) serves as an RNAidependent heterochromatin nucleation center at the endogenous locus and at an ectopic location (2). Although RNAi 